\title{
Effect of novel bright image enhanced endoscopy using blue laser imaging (BLI)
}

Authors

Institutions
Kazuhiro Kaneko', ${ }^{1,2}$, Yasuhiro Oono ${ }^{1}$, Tomonori Yano ${ }^{1}$, Hiroaki Ikematsu' ${ }^{1}$, Tomoyuki Odagaki ${ }^{1}$, Yusuke Yoda', Atsushi Yagishita ${ }^{1}$, Akihiro $\mathrm{Sato}^{3}$, Shogo Nomura ${ }^{3}$

${ }^{1}$ Department of Gastroenterology, Endoscopy Division, National Cancer Center Hospital East, Kashiwa, Japan

${ }^{2}$ Division of Science and Technology for Endoscopy and Surgery, National Cancer Center Hospital East, Kashiwa, Japan

${ }^{3}$ Exploratory Oncology Research \& Clinical Trial Center, National Cancer Center Hospital East, Kashiwa, Japan submitted $\quad$ 8. May 2014 accepted after revision 27. August 2014

\section{Bibliography}

DOI http://dx.doi.org/

10.1055/s-0034-1390707

Published online: 24.10 .2014

Endoscopy International Open 2014; 02: E212-E219

(c) Georg Thieme Verlag KG Stuttgart · New York

E-ISSN 2196-9736

\section{Corresponding author}

\section{Kazuhiro Kaneko, MD PhD}

Department of

Gastroenterology

Endoscopy Division

National Cancer Center Hospital

\section{East}

Kashiwanoha 6-5-1

Kashiwa

Chiba 277-8577

Japan

Fax: +81-4-71314724

kkaneko@east.ncc.go.jp
Background and study aims: The novel method of image-enhanced endoscopy (IEE) named blue laser imaging (BLI) can enhance the contrast of surface vessels using lasers for light illumination. BLI has two IEE modes: high contrast mode (BLI-contrast) for use with magnification, and bright mode (BLI-bright), which achieves a brighter image than BLI-contrast and yet maintains the enhanced visualization of vascular contrast that is expected for the detection of tumors from a far field of view. The aim of this study is to clarify the effect of BLI-bright with a far field of view compared to BLI-contrast and commonly available narrow-band imaging (NBI).

Patients and methods: Patients with neoplasia, including early cancer in the pharynx, esophagus, stomach, or colorectum, were recruited and underwent tandem endoscopy with BLI and NBI systems. Six sets of images of the lesions were cap-

\section{Introduction \\ $\nabla$}

In recent years, image-enhanced endoscopy (IEE), such as narrow-band imaging (NBI) or flexible spectral imaging color enhancement (FICE), has been used to improve visualization of the vascular patterns in the surface of the mucosa for enhanced diagnosis, such as histological diagnosis $[1,2]$, distinguishing between neoplasia and nonneoplasia [3], and depth of infiltration [4].

NBI is a commonly used IEE method that enhances the visualization of the surface vascular pattern using optical filters against a xenon lamp that allows narrow-band light to pass at wavelengths of 415 and $540 \mathrm{~nm}$. NBI utilizes the wavelength-dependent absorption rate of hemoglobin and the scattering characteristics of tissue to enhance the visible vascular pattern. Short wavelength light, or blue light ( $415 \mathrm{~nm}$ ), is strongly absorbed by hemoglobin in the shallow depth of the mucosa that is permeated with surface blood vessels, and the long wavelength light $(540 \mathrm{~nm})$ goes tured with a changing observable distance from 3 to $40 \mathrm{~mm}$. Individual sets of images taken from various observable distances were assessed for visibility among BLI-bright, BLI-contrast, and NBI modes. The brightness and contrast of these images were also analyzed quantitatively.

Results: Of 51 patients, 39 were assessed. Image analysis indicated that only BLI-bright maintained adequate brightness and contrast up to $40 \mathrm{~mm}$ and had significantly longer observable distances compared to the other methods. Furthermore, BLI-bright enhanced the visualization of serious lesions infiltrating into deeper layers, such as esophageal lamina propria or gastric submucosal cancers.

Conclusions: BLI-bright will be a helpful tool for the far-field view with IEE in organs with wider internal spaces such as the stomach.

through to the middle depth of the mucosa and the middle-depth blood vessels. Shorter wavelength light can depict only the surface information on the mucosa such as surface blood vessels and surface structure patterns instead of the mixed information as seen with the deeper area visualized by white light (WL) illumination. NBI is also effective for detection of neoplasia in the head and neck region and esophagus [5]. In the stomach, non-magnifying NBI does not work for detection because it is too dark [6]. In colon polyp detection, NBI does not improve the adenoma detection rate during primary colonoscopy compared to results with WL imaging [7].

Recently, a new IEE method named "Blue Laser Imaging" (BLI) has been developed by FUJIFILM Corporation (Tokyo, Japan) [8]. The BLI system has a unique feature of illumination using two lasers and a white light phosphor to accomplish the visual enhancement of surface vessels and structures. One of the lasers, with a wavelength of $450 \mathrm{~nm}$, stimulates the phosphor to irradiate a 
white-color illumination, and the other laser, with a wavelength of $410 \mathrm{~nm}$, is used to enhance the blood vessels at shallow depth in the mucosa. The BLI system switches between WL imaging mode and BLI mode by changing the intensity of these lasers.

In this study, we evaluated a prototype of the BLI endoscopy system that is equipped for two BLI observation modes by changing the intensity balance of these two kinds of laser. One mode is designed for high-contrast imaging (BLI-contrast) to observe blood vessels in detail by near field or magnified view. The other (BLIbright) is designed for a brighter image and yet maintains the vascular contrast that is expected with use of a far field of view; this may help to detect lesions in screening endoscopy. These two BLI modes and the WL mode can be easily and instantly switched by pressing a button on the handle of the scope.

BLI with magnification can predict histopathological diagnosis and invasion depth with well-matched diagnostic effectiveness compared with NBI magnification [9]. However, there has been no report that evaluated the effect regarding observable distance in BLI-bright mode. It is difficult to evaluate the efficacy between BLI and NBI because visibility is affected by the location of the lesion, observable distance, features of the lesion, and so on.

In this study, we evaluated the clinical utility of the BLI-bright mode by measuring the observable distance from lesions and comparing this to similar data from the BLI-contrast and commonly available NBI techniques. We performed endoscopic examinations using BLI and NBI systems in a tandem manner in patients who had neoplastic lesions containing early cancers in the pharynx, esophagus, stomach, or colorectum. With this process, we observed the same lesion on the same day with both endoscopy systems. Then, brightness and contrast in these images of the lesions were evaluated quantitatively according to a general formula.

\section{Patients and methods}

$\nabla$

\section{Patients}

Patients who had scheduled endoscopic examinations for either suspected neoplastic lesions including adenoma, or endoscopic resection for intramucosal cancer in the middle or lower pharynx, esophagus, stomach, or left colon and rectum from February to August 2011 at the National Cancer Center Hospital East in Japan were assigned to this study. They were all over 20 years of age, and their Eastern Cooperative Oncology Group (ECOG) performance status was 0 or 1 . Patients who had received surgical resection, chemotherapy, or radiotherapy were excluded from this study. All patients gave written informed consent, after clear and thorough explanation, to undergo tandem endoscopy in a day, after the study was approved by the institutional review board of our hospital. This study has been registered in the University Hospital Medical Information Network Clinical Trials Registry (UMIN-CTR) as study \#UMIN (ID: 000004983).

\section{BLI endoscope system}

The prototype of the BLI laser illumination endoscopic system developed by FUJIFILM was used in this study. The system consists of the VP-4450HD processor, the laser light source, and special scope series. The light source has two lasers; one of them with a wavelength of $450 \mathrm{~nm}$ to stimulate the phosphor in the tip of the special scope to irradiate white-color illumination that is similar to that of a xenon lamp, and the other laser with a wavelength of $410 \mathrm{~nm}$ to enhance the visualization of blood vessels.
This BLI system has three observation modes: WL mode, BLI-contrast, and BLI-bright. The outward appearance of the visibility in WL mode is the same as that seen with a conventional xenon light. BLI-contrast is accomplished with a strong $410 \mathrm{~nm}$ laser intensity ratio for a high contrast image of blood vessels in the shallow depth of the mucosa. BLI-bright is designed for a brighter image and yet maintains vascular contrast with a suitable intensity balance of these lasers. While BLI-contrast is made to observe blood vessels in detail with a near field or magnified view, the novel feature of BLI-bright is its use with a far field of view that may help in the detection of lesions in screening endoscopy. These three modes can be easily and instantly switched by pressing a button on the handle of the scope.

\section{Design of the clinical trial}

This was the first in-human clinical trial using a novel BLI prototype. This study was a prospective, comparative study using tandem endoscopic observation with both BLI and NBI. The BLI system was a prototype of the BLI endoscopy system (FUJIFILM, Tokyo, Japan), and the NBI system generally used in this study was the CV-260SL processor and CLV-260SL light source with a GIFH260Z or CF-H260AZI scope (Olympus, Tokyo, Japan).

Patients underwent tandem repeated endoscopic examinations with both the BLI system and NBI system on the same day. For those patients who were scheduled for endoscopic resection, endoscopy with the BLI system was performed first, and then endoscopy with NBI was performed, because the existence and location of the individual lesions had already been confirmed by initial endoscopy in our hospital. For those patients who had suspected neoplastic lesions in the left side colon and rectum, colonoscopy with the NBI system was performed first, and then colonoscopy with BLI was performed. When no neoplastic lesions, including early cancer, were found at the first colonoscopy, the patient's enrollment was terminated without second colonoscopy. Only one lesion was selected for each patient for assessment even though there were several lesions. Images were acquired at distances of approximately 40,30,20, and $10 \mathrm{~mm}$ from the lesion site without magnification, and at a distance of about 5 and $3 \mathrm{~mm}$ with magnification.

The following method was used to measure the distance between the lesion and the endoscope. The depths of field of the BLI prototype scope, GIF-H260Z, and CF-H260AZI were 6-100 mm, 7$100 \mathrm{~mm}$, and $7-100 \mathrm{~mm}$ without magnification, and $2-3 \mathrm{~mm}$, $1.5-3 \mathrm{~mm}$, and $2-3 \mathrm{~mm}$ with magnification, respectively. At first, the image of the nearest position from the surface of the mucosa where a clear image could be obtained without magnification, i.e. just a few millimeters from the minimum depth of field of each scope, was acquired and was defined as the image of "10 mm". Then the scope was pulled out by hand in steps of $10 \mathrm{~mm}$ and the images acquired were defined as $20 \mathrm{~mm}, 30 \mathrm{~mm}$, and $40 \mathrm{~mm}$, respectively. Then, with the same technique, images from distances of about $5 \mathrm{~mm}$ with medium magnification, and from about $3 \mathrm{~mm}$ with full magnification were obtained, respectively. When the procedure was performed with the BLI system, images of BLI-bright and BLI-contrast were acquired consequently by switching between these modes. Among the BLI and NBI systems, the lesion was defined to be visible as a brownish area in individual organs because there has been no official evaluation method regarding far-field observation in IEE. The brownish area was defined as accumulation of neovascularity in the lesion [10]. When the demarcation line of the brownish area could not be recog- 

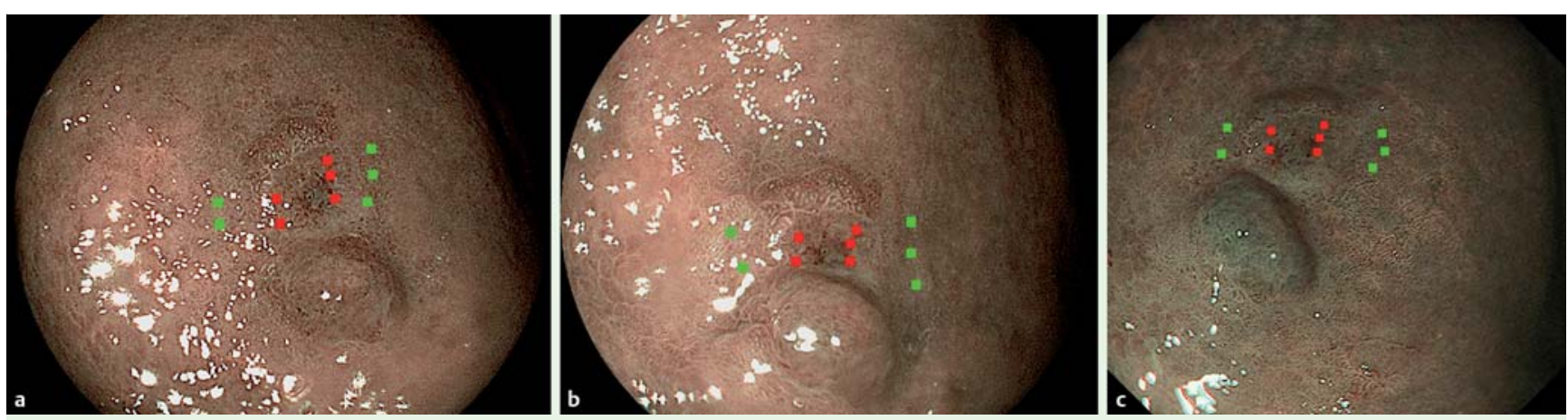

Fig. 1 Example of the sampling areas for image analysis observed by a BLI-bright, $\mathbf{b}$ BLI-contrast, $\mathbf{c}$ NBI. Red points are sampling areas on the lesion, and green points are those on the normal mucosa.

nized, the judgment was "negative" even though the lesion was detected by the morphological change.

All images were assessed at a later date by a single endoscopist (KK). Images were displayed on a monitor, in the order of NBI, BLI-bright, and then BLI-contrast, and in descending order of observation distance from $40 \mathrm{~mm}$ to $3 \mathrm{~mm}$, to establish whether it was "possible to distinguish regions exhibiting neoplastic changes" as a brownish area. The farthest observation distance of the images where such regions could be distinguished was defined as the "observable distance". Each lesion was confirmed by histopathologic analysis from the endoscopic resection or biopsy specimens according to the Japanese classification for cancer of the head and neck [11], esophagus [12], stomach [13], and colon and rectum [14]. From resected specimens, the depth of infiltration in individual lesions was evaluated, such as epithelial (EP), lamina propria mucosae (LPM), and muscularis mucosa (MM) in the esophagus; mucosal (M) and submucosal (SM) cancer in the stomach; and adenoma, mucosal (M), and submucosal (SM) cancer in the colorectum.

\section{Quantitative evaluation of image data}

Images taken from the stomach were used for the quantitative evaluation of brightness and contrast. The image was selected with the following conditions: 1) images were available for all conditions (BLI-bright/BLI-contrast/NBI, distance of 40/30/20/ $10 \mathrm{~mm}), 2$ ) the imaging angle was a downward view, 3) the lesion was distinguished without magnification in at least one of the conditions, and 4) the periphery of the lesion as a brownish area was clearly distinguishable from normal mucosa.

Five sampling areas near the periphery of the lesion, and five corresponding sampling areas on the surrounding normal mucosa were selected manually in each image ( $\bullet$ Fig. 1 ). Each sampling area included 100 pixels $(10 \times 10$ pixels), and was selected as the same position on the mucosa, avoiding the specular reflection area in the set of images of the same lesion.

The brightness Ym was defined as an average of the luminance values in the sampling areas on the surrounding mucosa according to the formula (1) as shown below [15]:

$$
\mathrm{Ym}=0.30 \times \mathrm{R}+0.59 \times \mathrm{G}+0.11 \times \mathrm{B} \ldots(1)
$$

where R, G, and B indicate average signal intensities of red, green and blue in the sampling area, respectively.

Contrast was defined by the following formula (2):

$$
\text { Contrast }=(\mathrm{Ym}-\mathrm{Yv}) / \mathrm{Ym}(2)
$$

where Yv is the average brightness in the sampling area on the lesion, and Ym is the average brightness in the corresponding sampling area on the surrounding mucosa. The brightness and contrast of each image were defined as an average of each value from the five sampling areas.

\section{Statistical analysis}

Patient characteristics and the observable distance as a result of evaluation were summarized according to the disease site. Observable distances for either BLI-contrast or NBI were compared with those of BLI-bright using the Wilcoxon signed-rank test. All statistical tests were performed with SPSS version 11 (SPSS Inc, Chicago, IL, United States). All P-values were reported as two-sided without adjusting for the multiple testing problem.

\section{Results \\ $\nabla$}

\section{Patient characteristics}

After 51 patients had been recruited, these patients underwent the first endoscopy. Eleven patients were removed from analysis of the study after the first endoscopy because no lesion was found, and 40 patients immediately underwent the second examination. Of the 40 lesions, 39 were histologically evaluated for endoscopic resection and the remaining one lesion was evaluated by biopsy specimen. One stomach lesion could not be distinguished without magnification, neither with BLI nor with NBI. This case was diagnosed by histopathology as signet ring cell carcinoma. Hence, this was regarded as indistinguishable and was excluded from subsequent analysis. Finally, a total of 39 lesions from 39 patients were analyzed in this study ( Table 1 ).

\section{Observable distance among BLI-bright, BLI-contrast and $\mathrm{NBI}$ images}

An example set of images for the colon adenoma case is shown in - Fig. 2. In this case, the whole lesion could be distinguished at up to a distance of $40 \mathrm{~mm}$ in the series of BLI-bright images. In the series of BLI-contrast images and NBI images, the clarity of the lesion decreased as the observation distance increased; the lesion could not be distinguished at an image distance of $40 \mathrm{~mm}$. Therefore, the observation distances for BLI-bright, BLI-contrast, and NBI in this case were read as 40,20 , and $10 \mathrm{~mm}$, respectively. Since this lesion was composed of flat and nodular portions, the nodule was visible at $40 \mathrm{~mm}$ in all three modes. However, the whole lesion composed of flat and nodular portions was visible in BLI-bright alone. Visualization of the flat portion from a far field of view has clinical impact.

- Table 2 shows the number of cases by individual observable distances, and Table 3 shows the mean observable distances. 
Table 1 Analyzed patient characteristics $(n=39)$

\begin{tabular}{|l|l|}
\hline Age & 71.0 \\
\hline Mean, years & $49-85$ \\
\hline Range, years & \\
\hline Sex, $n(\%)$ & $32(82 \%)$ \\
\hline Men & $7(18 \%)$ \\
\hline Women & \\
\hline Location, $n(\%)$ & $3(8 \%)$ \\
\hline Pharynx & $8(21 \%)$ \\
\hline Esophagus & $(2)$ \\
\hline SCC EP & $(4)$ \\
\hline LPM & $(2)$ \\
\hline Adenocarcinoma & $14(36 \%)$ \\
\hline Stomach & $(9)$ \\
\hline M & $(5)$ \\
\hline SM & $14(36 \%)$ \\
\hline Colorectum & \\
\hline
\end{tabular}

EP, epithelium; LPM, lamina propria mucosae; M, mucosa; SCC, squamous cell carcinoma; SM, submucosa.

The overall mean observable distances of BLI-bright, BLI-contrast, and NBI were $31.3 \mathrm{~mm}, 24.7 \mathrm{~mm}$, and $23.8 \mathrm{~mm}$, respectively. There was a significant difference for BLI-bright compared to BLI-contrast and NBI $(P<0.01$, and $P<0.01$, respectively).

For both BLI and NBI, the observable distance was different depending on the organ where the lesion was located. The longest distance was seen in the pharynx, and the shortest was in the stomach. An example of a set of images in the pharynx, esopha- gus, and stomach, when the observable distances were $40 \mathrm{~mm}$ and $20 \mathrm{~mm}$, is shown in $\bullet$ Fig. 3. An example of colon distances is shown in Fig. $\bigcirc$ 2. The maximum observable distance in the stomach for BLI-bright $(26.8 \mathrm{~mm})$ was significantly longer than that for NBI ( $16.4 \mathrm{~mm}, P=0.03)$. There were no significant differences in maximum observable distances for lesions in other organs and IEE methods.

- Fig. 4 shows that the observable distances depended on the depth of infiltration of histopathological findings in the esophagus and stomach. Because of the limited number of cases in our study, no significant statistical difference could be described, however, BLI-bright showed that observable distances tended to be longer in esophageal cases of the LPM than in the EP, and were longer in gastric SM cases than in mucosal cases.

\section{Quantitative evaluation}

To confirm the results from the endoscopist's assessments, quantitative evaluation of image data was conducted using representative cases in the stomach because the differences between BLIbright and other methods were the most pronounced in the stomach lesion cases. Seven cases, which could be estimated in all observable distances from $40 \mathrm{~mm}$ to $3 \mathrm{~mm}$, were selected from the 13 stomach cases that could be used for the image analysis according to the criteria. Mean observable distances from the selected seven cases were $37.1 \mathrm{~mm}, 17.6 \mathrm{~mm}$, and $22.1 \mathrm{~mm}$ in BLI-bright, BLI-contrast, and NBI, respectively.

The contrast curve depending on the observable distance is shown in Fig. 5 a. It is normalized at $10 \mathrm{~mm}$ of observable dis-

\begin{tabular}{|c|c|c|c|c|c|}
\hline & $\begin{array}{l}\text { All } \\
(n=39)\end{array}$ & $\begin{array}{l}\text { Pharynx } \\
(n=3)\end{array}$ & $\begin{array}{l}\text { Esophagus } \\
(n=8)\end{array}$ & $\begin{array}{l}\text { Stomach } \\
(n=14)\end{array}$ & $\begin{array}{l}\text { Colorectum } \\
(n=14)\end{array}$ \\
\hline \multicolumn{6}{|l|}{ BLI-bright } \\
\hline $40 \mathrm{~mm}$ & 21 & 2 & 5 & 6 & 8 \\
\hline $30 \mathrm{~mm}$ & 9 & 1 & 1 & 3 & 4 \\
\hline $20 \mathrm{~mm}$ & 4 & 0 & 1 & 1 & 2 \\
\hline $10 \mathrm{~mm}$ & 1 & 0 & 0 & 1 & 0 \\
\hline $5 \mathrm{~mm}$ & 4 & 0 & 1 & 3 & 0 \\
\hline $3 \mathrm{~mm}$ & 0 & 0 & 0 & 0 & 0 \\
\hline \multicolumn{6}{|c|}{ BLI-contrast } \\
\hline $40 \mathrm{~mm}$ & 10 & 2 & 2 & 1 & 5 \\
\hline $30 \mathrm{~mm}$ & 10 & 1 & 2 & 2 & 5 \\
\hline $20 \mathrm{~mm}$ & 9 & 0 & 2 & 5 & 2 \\
\hline $10 \mathrm{~mm}$ & 7 & 0 & 2 & 3 & 2 \\
\hline $5 \mathrm{~mm}$ & 2 & 0 & 0 & 2 & 0 \\
\hline $3 \mathrm{~mm}$ & 1 & 0 & 0 & 1 & 0 \\
\hline \multicolumn{6}{|l|}{ NBI } \\
\hline $40 \mathrm{~mm}$ & 9 & 2 & 0 & 1 & 6 \\
\hline $30 \mathrm{~mm}$ & 10 & 1 & 3 & 3 & 3 \\
\hline $20 \mathrm{~mm}$ & 9 & 0 & 4 & 2 & 3 \\
\hline $10 \mathrm{~mm}$ & 7 & 0 & 1 & 4 & 2 \\
\hline $5 \mathrm{~mm}$ & 4 & 0 & 0 & 4 & 0 \\
\hline $3 \mathrm{~mm}$ & 0 & 0 & 0 & 0 & 0 \\
\hline
\end{tabular}

\begin{tabular}{|llllll|l|}
\hline & BLI-bright & BLI-contrast & Pvalue $^{\mathbf{1}}$ & NBI & Pvalue $^{\mathbf{2}}$ & $\begin{array}{l}\text { Table 3 } \\
\text { tance }\end{array}$ \\
\hline All & 31.3 & 24.7 & $<0.01$ & 23.8 & $<0.01$ & \\
\hline Pharynx & 36.7 & 36.7 & 1.00 & 36.7 & 1.00 & \\
\hline Esophagus & 31.9 & 25 & 0.13 & 22.5 & 0.06 & \\
\hline Stomach & 26.8 & 17.4 & 0.04 & 16.4 & 0.03 & \\
\hline Colorectum & 34.3 & 29.3 & 0.09 & 29.3 & 0.17 & \\
\hline
\end{tabular}

1 P value between BLI-bright vs. BLI-contrast.

2 value between BLI-bright vs. NBI. 

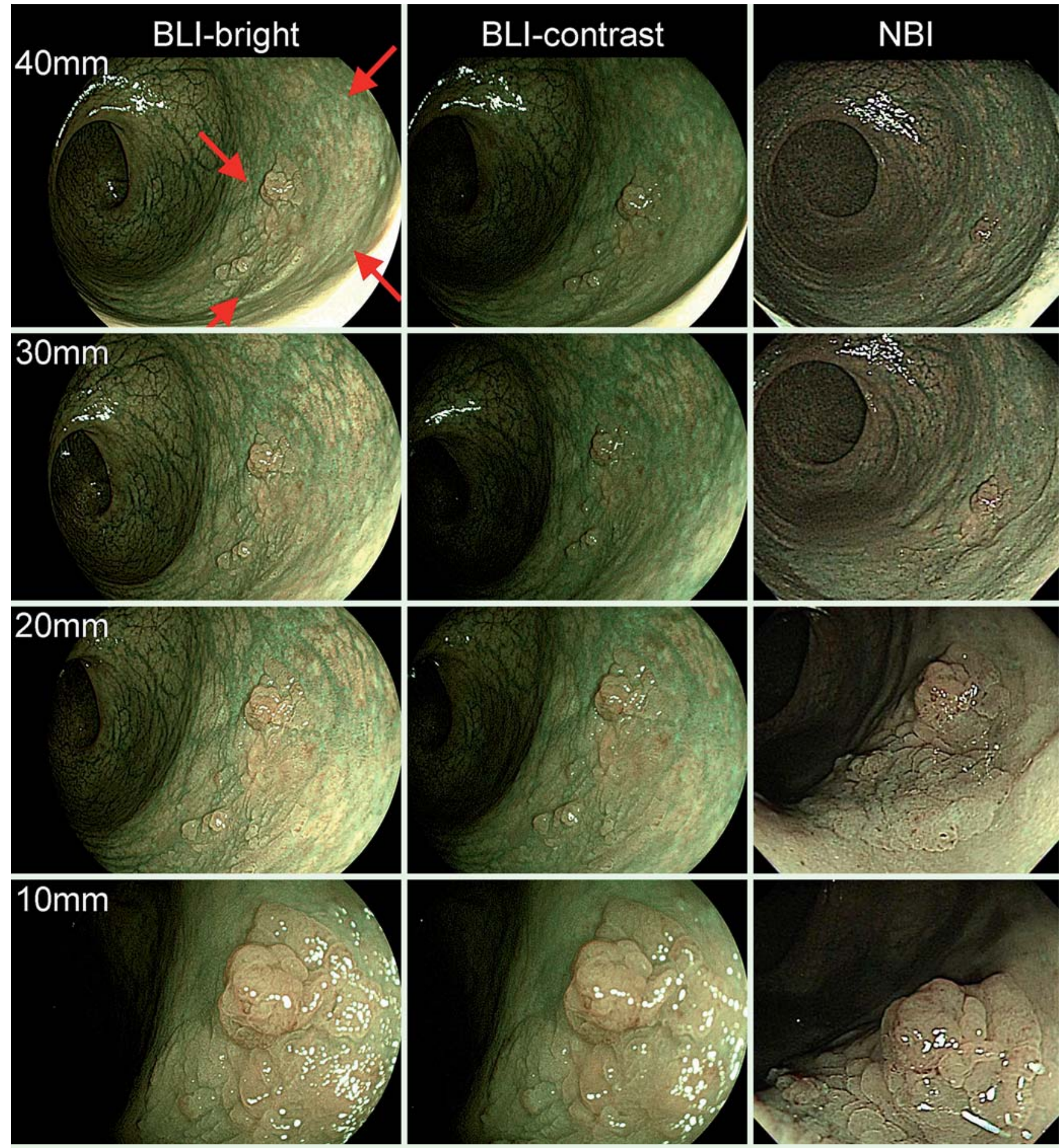

Fig.2 Example of the distance/IEE mode-dependent images of the same case. Left: BLI-bright, middle: BLI-contrast, right: NBI. Images are shown at $40 \mathrm{~mm} /$ $30 \mathrm{~mm} / 20 \mathrm{~mm} / 10 \mathrm{~mm}$ observation distances. Red arrows indicate the lesion. One nodule in the lesion was visible in three modes at $40 \mathrm{~mm}$ observation distance, however, the whole lesion, composed of flat and nodular portions, was visible in BLI-bright alone.

tance. While the contrast curve for BLI-bright and BLI-contrast is stable from $10 \mathrm{~mm}$ to $40 \mathrm{~mm}$, the contrast curve for NBI decreases as the distance increases to more than $20 \mathrm{~mm}$.

The brightness curve dependent on observation distance is shown in Fig. $\mathbf{5}$ b. The brightness curve for each mode degraded depending on the observable distance. Compared to the brightness curve of BLI-bright or NBI, the degradation of BLI-contrast decreased rapidly at distances greater than $30 \mathrm{~mm}$. This means that the image of BLI-contrast became darker more rapid- ly with increasing distance than it did in the other modes, making it difficult to find the lesions from a distance.

Longer observation distances made it difficult to distinguish the lesions by NBI because of the degradation of contrast, although the image looked bright. Therefore, the BLI-bright mode provided the best image to distinguish lesions in this situation. 


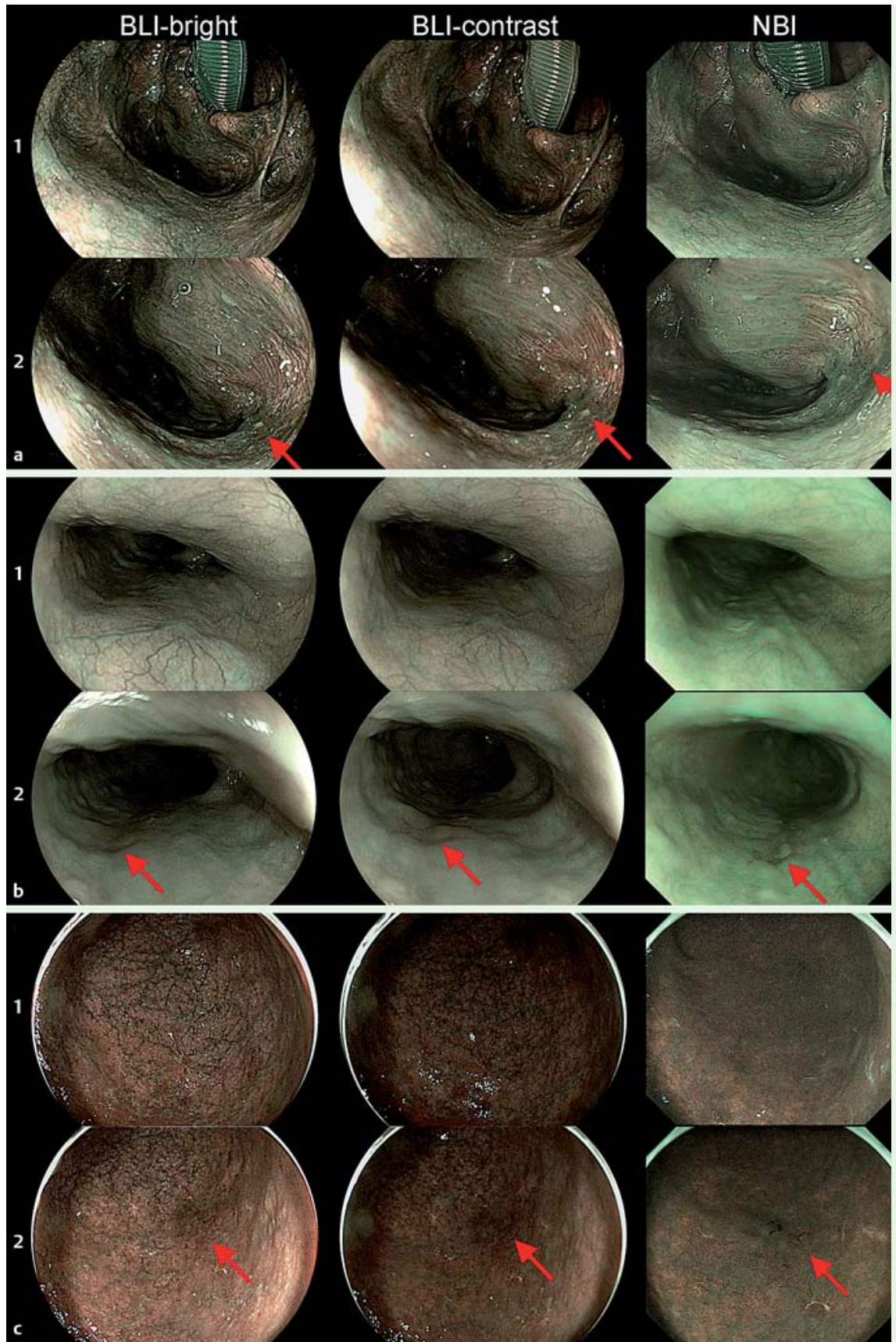

Fig. 3 Endoscopic images of lesions in the a pharynx, b esophagus, c stomach, illuminated by BLIbright (left), BLI-contrast (middle), NBI (right) from observation distances of 1) $40 \mathrm{~mm}$, 2) $20 \mathrm{~mm}$. Red arrows indicate the lesion.

\section{Discussion}

$\nabla$

IEE is an effective method to detect neoplasia, distinguish neoplasia from non-neoplasia, and to diagnose precursor or early cancer. NBI uses narrow band light by filtering a xenon light source. It is suitable to visualize microvessels, however, the image becomes dark when it is used for a far field of view. The new IEE "BLI" device is a unique imaging method using laser light illumination. Because of this unique method, it is able not only to achieve a high contrast image for the enhancement of surface information but also to achieve a bright image.

This study revealed analytically the relationship of brightness, contrast, and observable distance for BLI-bright, BLI-contrast, and NBI in neoplasia of the human gastrointestinal tract. From the assessment by an endoscopist, it was revealed that BLIbright had significantly longer observable distances than the other methods. It was also shown that BLI-bright mode had sig- nificantly longer observable distances than BLI-contrast mode as seen in the same system by changing the intensity balance of the lasers.

Examination of the endoscopic images showed that they became darker in wider space locations. A part of the illuminated light in the gastrointestinal tract reflects off the surface of the mucosa, increasing the overall illumination in the location. However, while this is particularly effective in a smaller space such as the pharynx, in a wider area such as the stomach, the reflected illumination diffuses more readily and provides much less benefit.

The statistical results corroborated this aspect. All cases in the pharynx could be distinguished from 30 or $40 \mathrm{~mm}$ by all methods. However, a significant difference between BLI-bright and the other methods was seen in the stomach, and an intermediate difference was seen in the colorectum and esophagus. Therefore, it can be said that BLI-bright will most benefit observations done in the stomach. Owing to the limited number of cases, it is un- 

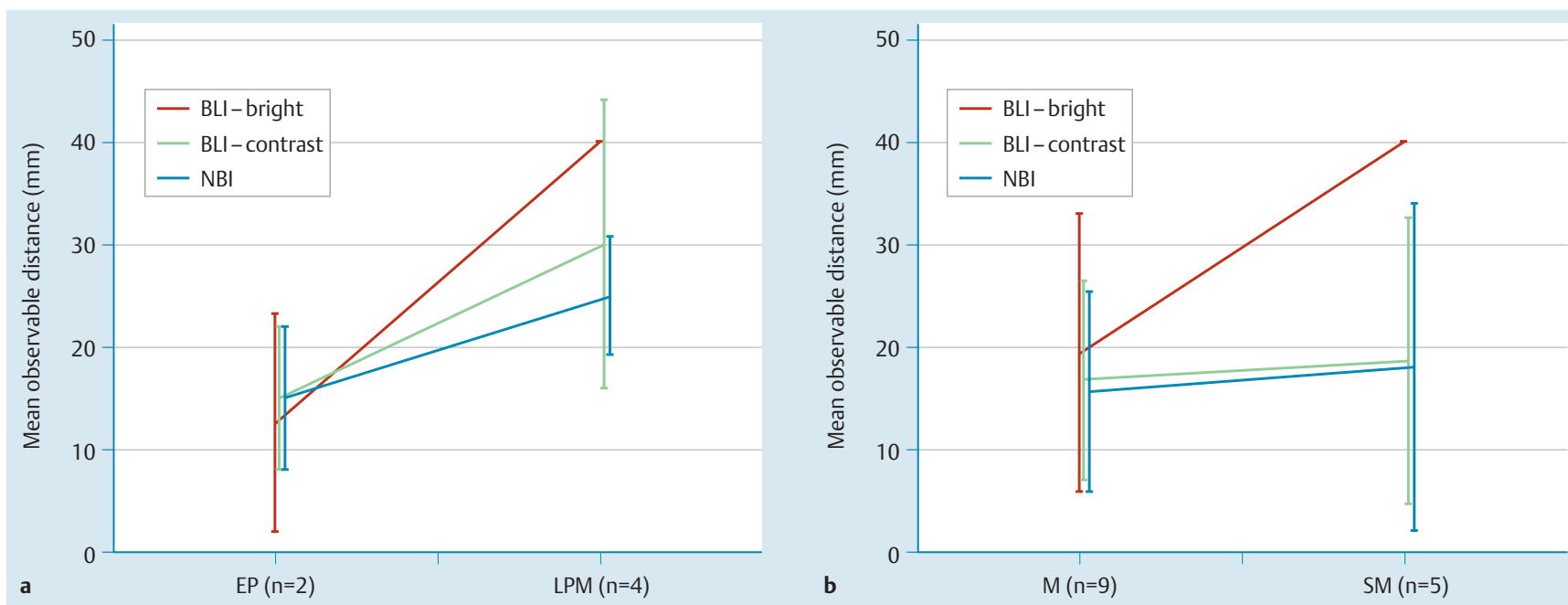

Fig.4 Average observable distances depending on the characteristics of each lesion. a EP, epithelial cancer; LPM, cancer infiltrating into the lamina propria mucosa; b M, mucosal cancer; SM, submucosal cancer.
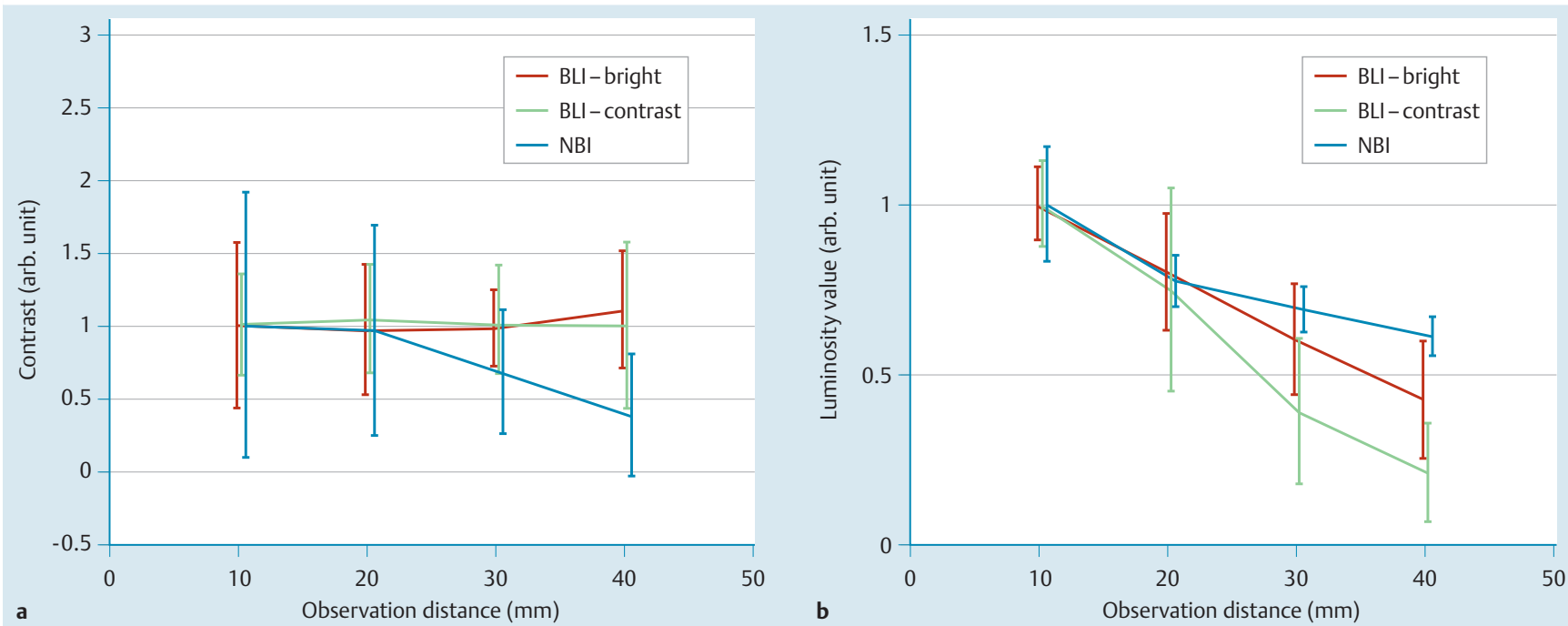

Fig. 5 a Contrast, and $\mathbf{b}$ brightness of BLI-bright, BLI-contrast, and NBI depending on the observation distances calculated from image analysis. Each line is normalized by the average value at $10 \mathrm{~mm}$.

clear whether it can be more effective than the other methods in the colorectum and esophagus; however, BLI-bright appears effective in the detection of lesions in these organs based on the results of this pilot study.

It was shown that visibility changes according to the characteristics of the lesion. It seems that BLI-bright has a longer observable distance than does NBI in cases where the lesions infiltrate into deeper layers, such as esophageal LPM or gastric SM cancers, rather than in cases with a low degree of differentiation. In our previous study, microvessel density had a correlation with tumor infiltration in esophageal squamous cell carcinomas [16]. Because the number and diameter of microvessels on the surface of the tumor increased according to invasion depth, we hypothesized that esophageal LPM or gastric SM cancers would be visible as a brownish area in BLI-bright from the far field of view. BLIbright might be able to detect slightly infiltrated tumors more effectively than would the other modes, and this capability is critical for establishing a correct diagnosis.

The visibility of lesions can usually be described by the relationship between the brightness and contrast of images. In general, brightness is needed to detect the color changes of the lesions, and contrast is needed to identify the demarcation of the lesion from normal mucosa. The light intensity of both systems was controlled via the automatic iris by default. Therefore, both brightness and contrast are needed to maintain effective visibility. Stable contrast such as that from the BLI-bright and BLI-contrast seen in Fig.5a, is desired. Contrast is independent of brightness when the illumination intensity is suitable, but when the illumination is too weak, the contrast degrades because the noise of the sensor device becomes relatively strong compared to the signal from the object. Even though the image brightness can be adjusted by auto-exposure or image processing, degradation of contrast may still be present. The brightness curve is determined by the balance between distance-dependent degradation, auto-light control function, and other adjustment functions of the system such as the auto-exposure function or image processing. The differences in these functions between the BLI and NBI systems caused the differences between the luminosity curves of BLI-bright and NBI as seen in $\bullet$ Fig. $\mathbf{5}$ b.

The visibility provided by BLI-contrast mode was limited in the far field of view owing to the lack of brightness as shown in - Fig. $\mathbf{5 b}$ b, although the contrast curve remained stable as seen 
in Fig. 5 a. Alternatively, the contrast of the commonly available NBI, as seen in $\bullet$ Fig. 5 a, decreased when the observation distance was more than $20 \mathrm{~mm}$. BLI-bright had a good balance of both contrast and brightness to allow for the detection of lesions from the far field of view. Both BLI-bright and BLI-contrast mode in this prototype seemed to have the potential to improve the visibility through adjustments because the observable distance was limited by the image brightness, although the contrast was stable up to $40 \mathrm{~mm}$ in both modes of BLI.

The most important goal of endoscopic screening is to enable the chance to identify an early cancer or precursor. In conventional endoscopy, the chance or motive of detection for neoplasia relies on the reddish areas, irregular mucosa, and elevations and depressions. In contrast, IEE lesions are detected through observation of a brownish area. However, magnified observation of suspected lesions is required in IEE, apart from pharyngeal and esophageal cancers. Because the target lesion can be visible in BLI-bright without magnification from the far field of view, we suggest that BLI-bright might be used as a new modality for cancer screening.

There were some limitations in our study. It was performed in a single institute with a small number of cases, and the observable distance among the three modes was evaluated by a single endoscopist. Observation became feasible at a distance a few millimeters short of $10 \mathrm{~mm}$ without magnification, demonstrating that the $10-\mathrm{mm}$ distance could serve as a basis. However, images were obtained at distances of $20 \mathrm{~mm}, 30 \mathrm{~mm}$, and $40 \mathrm{~mm}$ to minimize the observational error, with the scope withdrawn manually in increments of $10 \mathrm{~mm}$. Physical measures, such as forceps, which require precise placement, were not used, given the potential risk of hemorrhage through their contact with the lesion and the greater patient burden due to prolongation of the study time. The endoscopist (KK) had been informed about the lesions that needed to be assessed. Therefore, a "detectable" lesion was defined as a brownish area distinguishable in each image evaluated, instead of a tumor lesion as detected based on its morphological changes. Furthermore, it was necessary to perform final diagnosis and endoscopic treatment during NBI endoscopy used generally. Therefore, when endoscopy with a BLI system was performed first, it was followed by NBI endoscopy. The fact that the order of the images to be displayed was fixed under these circumstances might be limited as biased estimates of performance. This study was conducted using the commonly available NBI device because the new-generation NBI, which provides brighter imaging than the one used in this study, only became available after study registration. However, the aim of this present study was a preliminary performance evaluation using a novel endoscope system, with a first in-human clinical trial of this type. We feel that IEE with brighter technology has a greater potential for benefit in screening procedures than do current methods, and would be useful in clinical practice. Hence, performance assessment with a large scale clinical trial regarding the detection of neoplasia with this new BLI-bright mode would be desirable.

\section{Conclusion}

$\nabla$

The BLI system is a novel IEE using laser illumination that enables operators to change the brightness and contrast just by changing the laser intensity. BLI-bright mode gives the ability to capture a high-contrast image from a long distance, and BLI-contrast mode allows for magnified images. These two modes will facilitate both the detection of suspected lesions and the making of accurate diagnoses with magnified IEE images, especially in organs with wide internal spaces such as the stomach.

Competing interests: This author discloses the following: K. Kaneko received grant support and performed a clinical trial using a borrowed prototype endoscope system from Fujifilm Corporation, Tokyo, Japan. The remaining authors disclose no conflicts.

\section{Acknowledgments}

\section{$\nabla$}

This work was supported in part by the National Cancer Center Research and Development Fund, 23-A-15 (group 4), a fund for developing research regarding endoscopy in early phase (K. Kaneko).

\section{References}

1 Katagiri A, Fu KI, Sano Y et al. Narrow band imaging with magnifying colonoscopy as diagnostic tool for predicting histology of early colorectal neoplasia. Aliment Pharmacol Ther 2008; 27: 1269-1274

2 Yoshida N, Naito Y, Kugai $M$ et al. Efficacy of magnifying endoscopy with flexible spectral imaging color enhancement in the diagnosis of colorectal tumors. J Gastroenterol 2011; 46: 65 - 72

3 Horimatsu T, Sano Y, Kaneko $K$ et al. Relationship between MVD and meshed-capillaries using magnifying NBI colonoscopy in colorectal precursor lesions. Hepatogastroenterology 2009; 56: 372 - 377

4 Ikematsu H, Matsuda T, Emura F et al. Efficacy of capillary pattern type IIIA/IIIB by magnifying narrow band imaging for estimating depth of invasion of early colorectal neoplasms. BMC Gastroenterol 2010; 10: 33

5 Muto M, Minashi K, Yano $T$ et al. Early detection of superficial squamous cell carcinoma in the head and neck region and esophagus by narrow band imaging: a multicenter randomized controlled trial. J Clin Oncol 2010; 28: $1566-1572$

6 Ezoe $Y$, Muto $M$, Uedo $N$ et al. Magnifying narrowband imaging is more accurate than conventional white-light imaging in diagnosis of gastric mucosal cancer. Gastroenterology 2011; 141: 2017-2025

7 Ikematsu H, Saito Y, Tanaka S et al. The impact of narrow band imaging for colon polyp detection: a multicenter randomized controlled trial by tandem colonoscopy. J Gastroenterol 2012; 47: 1099-1107

8 Yoshida $N$, Yagi N, Inada Yet al. Ability of a novel blue laser imaging system for the diagnosis of colorectal polyps. Dig Endosc 2014; 26: 250 258

9 Yoshida $N$, Hisabe T, Inada Y et al. The ability of a novel blue laser imaging system for the diagnosis of invasion depth of colorectal neoplasms. J Gastroenterol 2014; 49: $73-80$

10 Muto M, Nakane M, Katada $C$ et al. Squamous cell carcinoma in situ at oropharyngeal and hypopharyngeal mucosal sites. Cancer 2004; 101: $1375-1381$

11 Japan Society for Head and Neck Cancer. Japanese guidelines for the treatment of head and neck carcinoma. Tokyo: Kanehara Shuppan Co; 2005

12 Japanese Esophageal Cancer Association. Japanese classification of esophageal cancer. 10th edn. Tokyo: Kanehara Shuppan Co; 2008

13 Japanese Gastric Cancer Association. Japanese classification of gastric cancer. 3rd edn. Tokyo: Kanehara Shuppan Co; 2010

14 Japanese Society for Cancer of the Colon and Rectum. Japanese guidelines for the treatment of colorectal carcinoma. Tokyo: Kanehara Shuppan Co; 2006

15 ITU-R Recommendation BT.709-5: Parameter values for the HDTV standards for production and international programme exchange. 04 2002: http://www.itu.int/rec/R-REC-BT.709/en

16 Kubota Y, Kaneko K, Konishi K et al. The onset of angiogenesis in a multistep process of esophageal squamous cell carcinoma. Front Biosci (Landmark Ed) 2009; 14: $3872-3878$ 